\title{
Plasma Biomarkers in Predicting Renal Recovery from Acute Kidney Injury in Critically III Patients
}

\author{
Marco Fiorentino ${ }^{a, b}$ Fadi A. Tohme ${ }^{a}$ Raghavan Murugan ${ }^{a, c} \quad$ John A. Kellum ${ }^{a, c}$ \\ ${ }^{a}$ The Center for Critical Care Nephrology, Department of Critical Care Medicine, University of Pittsburgh, Pittsburgh, \\ PA, USA; ${ }^{b}$ Department of Emergency and Organ Transplantation, Nephrology, Dialysis and Transplantation Unit, \\ University of Bari, Bari, Italy; ${ }^{\mathrm{C}}$ The CRISMA Center, Department of Critical Care Medicine, University of Pittsburgh, \\ Pittsburgh, PA, USA
}

\section{Keywords}

Acute kidney injury · Biomarkers · Renal recovery · Renal replacement therapy · Neutrophil gelatinase-associated lipocalin

\begin{abstract}
Background: Numerous studies have suggested a possible role for acute kidney injury (AKI) biomarkers in predicting renal recovery both before and after renal replacement therapy (RRT). However, definitions for recovery and whether to include patients dying but free of RRT may influence results. Objectives: To validate plasma neutrophil gelatinase-associated lipocalin (pNGAL) as a useful biomarker for predicting or improving the ability of clinical predictors alone to predict recovery following $\mathrm{AKI}$, including in our model plasma $\mathrm{B}$ type natriuretic peptide (pBNP) to account for cardiovascular events. Methods: We analyzed 69 patients enrolled in the Acute Renal Failure Trial Network study. pNGAL and pBNP were measured on days 2,7 , and 14 . We analyzed their predictive ability for subsequent recovery, defined as alive and independent from dialysis in 60 days. In sensitivity analyses, we explored changes in results with alternative definitions of recovery. Results: Twenty-nine patients (42\%) recovered from AKI. Neither pNGAL nor pBNP, alone or in combination, was accurate predictors of renal recovery-the best area un-
\end{abstract}

der the receiver-operating characteristics curve (AUC) was for $\mathrm{pNGAL}$ using the largest relative change (AUC 0.59, 95\% $\mathrm{Cl}$ 0.45-0.74). The best clinical model achieved superior performance to biomarkers (AUC 0.69, 95\% CI 0.56-0.81). The AUC was greatest $(0.75,95 \% \mathrm{Cl} 0.60-0.91)$ when pNGAL + pBNP on day 14 were added to the clinical model but this increase did not achieve statistical significance. However, integrated discrimination improvement analysis showed that the addition of pNGAL and pBNP on day 14 to the clinical model significantly improved the prediction of renal recovery $(p=0.008)$. Conclusions: pNGAL and pBNP can improve the accuracy of clinical parameters in predicting AKI recovery and a full model using biomarkers together with age achieved adequate discrimination.

๑) 2019 S. Karger AG, Basel

\section{Introduction}

While recovery after severe acute kidney injury (AKI) receiving renal replacement therapy (RRT) may still occur in some patients, a significant proportion of patients can show partial recovery or do not recover at all. Nonrecovery of renal function carries significant negative effects on quality of life and health-care costs $[1,2]$, prolonged hospitalization, increased risk for chronic comor-

\section{KARGER}

(c) 2019 S. Karger AG, Basel

E-Mail karger@karger.com

www.karger.com/bpu
John A. Kellum, MD, MCCM

Center for Critical Care Nephrology, Department of Critical Care Medicine

Bioengineering, Translational and Clinical Science Director

3347 Forbes Avenue, Suite 220, Pittsburgh, PA 15213 (USA)

E-Mail kellum@pitt.edu 
bidities, and mortality [3]. Recovery from AKI varied in several clinical trials due to several factors, such as differences in the study population and in the definition of recovery [4]. Hence, the ability to accurately predict the possibility of recovery after AKI would have significant impact in the clinical management of critically ill patients [5]. Clinical predictors could help physicians to predict renal recovery and to assess clinical decisions, such as defining the timing of initiation and/or discontinuation of RRT $[1,6]$. Several urinary and plasma biomarkers have been investigated in predicting AKI development and severity, but only few studies explored their ability in predicting renal recovery $[7,8]$. We previously tested plasma neutrophil gelatinase-associated lipocalin (pNGAL) in patients with community-acquired pneumonia and found that elevated neutrophil gelatinase-associated lipocalin (NGAL) levels were associated with renal nonrecovery [5]. We also reported results from the Biological Markers of Recovery for the Kidney study, where urinary biomarkers were evaluated and compared between patients who did or did not recover from AKI requiring RRT [9]: significant differences were found between the 2 groups and urinary hepatocyte growth factor and urinary NGAL improved clinical risk prediction for renal recovery [9].

In the present study, we sought to validate pNGAL as a useful biomarker for improving the ability of clinical predictors alone to predict recovery following AKI. Finally, decreased survival after AKI is strongly influenced by cardiovascular events [10]. We included plasma Btype natriuretic peptide ( $\mathrm{pBNP}$ ) to account for the cardiovascular component in our models.

\section{Materials and Methods}

\section{Study Design}

The present study was a prospective cohort study conducted as an ancillary analysis to the Acute Renal Failure Trial Network (ATN) study. ATN was a multicenter, prospective study that aimed to compare 2 different strategies of RRT in critically ill patients with AKI [11]. Briefly, patients with AKI and failure of at least one nonrenal organ or sepsis were randomized between November 2003 and July 2007 to receive either intensive treatment (intermittent hemodialysis and sustained low-efficiency dialysis provided 6 times per week, and continuous venovenous hemodiafiltration providing a total effluent flow rate of $35 \mathrm{~mL} / \mathrm{kg}$ of body weight per hour) or less intensive RRT (intermittent hemodialysis and sustained low-efficiency dialysis provided 3 times per week, and continuous venovenous hemodiafiltration providing a total effluent flow rate of $20 \mathrm{~mL} / \mathrm{kg}$ of body weight per hour) from 27 VA and university-affiliated medical centers. The present cohort includes patients with availability of plasma biomarkers (pNGAL and pBNP) at specific time points (day 2, 7, and 14). The analysis was based only on day 2 and 7 measurements for patients without samples on day 14. Patients with preexisting chronic kidney disease were excluded from the ATN study. The Institutional Review Boards at all participating sites approved the parent study, and written informed consent was obtained from all participants or their proxies.

\section{Data Collection and Laboratory Measurements}

Clinical records of participants were prospectively collected in order to analyze baseline clinical characteristics, renal function, causes of AKI, and severity of illness scores. Sepsis was defined according to International Sepsis Definitions recommendations [12]. The primary outcome was recovery from AKI, defined as survival and dialysis independence in 60 days.

Plasma NGAL measurement was performed using the Triage NGAL Assay (Alere Inc., San Diego, CA, USA); it is a fluorescencebased sandwich immunoassay, which measures pNGAL with a measurable range from 15 to $1,300 \mathrm{ng} / \mathrm{mL}$. After addition of the sample to the device, the filter separated blood cells from plasma and the analyzer displayed the results in about $15 \mathrm{~min}$. Plasma BNP testing was also performed on the Triage platform using a standard commercially available assay (Alere Triage BNP Test, Alere Inc.).

\section{Statistical Analyses}

We compared clinical characteristics between patients who recovered and patients who failed to recover from AKI in 60 days. Continuous data were presented as mean and SD and compared with Student $t$ test or Mann-Whitney test, as appropriate. Categorical data were reported as proportions and compared using the chi-square or Fisher exact test. Plasma biomarkers (pNGAL, alone or in combination with pBNP, as markers of patient recovery) were measured at specific time points (days 2, 7 , and 14 after enrollment); furthermore, we analyzed the largest relative change between 2 and 7 or day 14 . We examined the association between each marker and renal recovery with logistic regression models and generated area under the receiver-operating characteristics (ROC) curves (AUC) to analyze the accuracy in predicting recovery. We used clinical risk prediction models identified in this population in our previous publication [9], based on age, Acute Physiology and Chronic Health Evaluation (APACHE II) score and Charlson comorbidity index; then, we added each biomarker model individually to the best clinical model and compared the composite models using AUC analysis. We performed sensitivity analyses using alternative definitions of recovery. Finally, we calculated the integrated discrimination improvement to examine the improvement in reclassification with the biomarkers compared to the clinical model alone. Analyses were performed using SAS software version 9.4 (SAS Institute, Cary, NC, USA), with statistical significance set at $p<0.05$.

\section{Results}

\section{Baseline Patient Characteristics and Outcomes}

A total of 69 patients had complete clinical data available and 29 patients (42\%) achieved the primary end- 
Fig. 1. Flow chart of study population. pNGAL, plasma neutrophil gelatinase-associated lipocalin; pBNP, B-type natriuretic peptide.

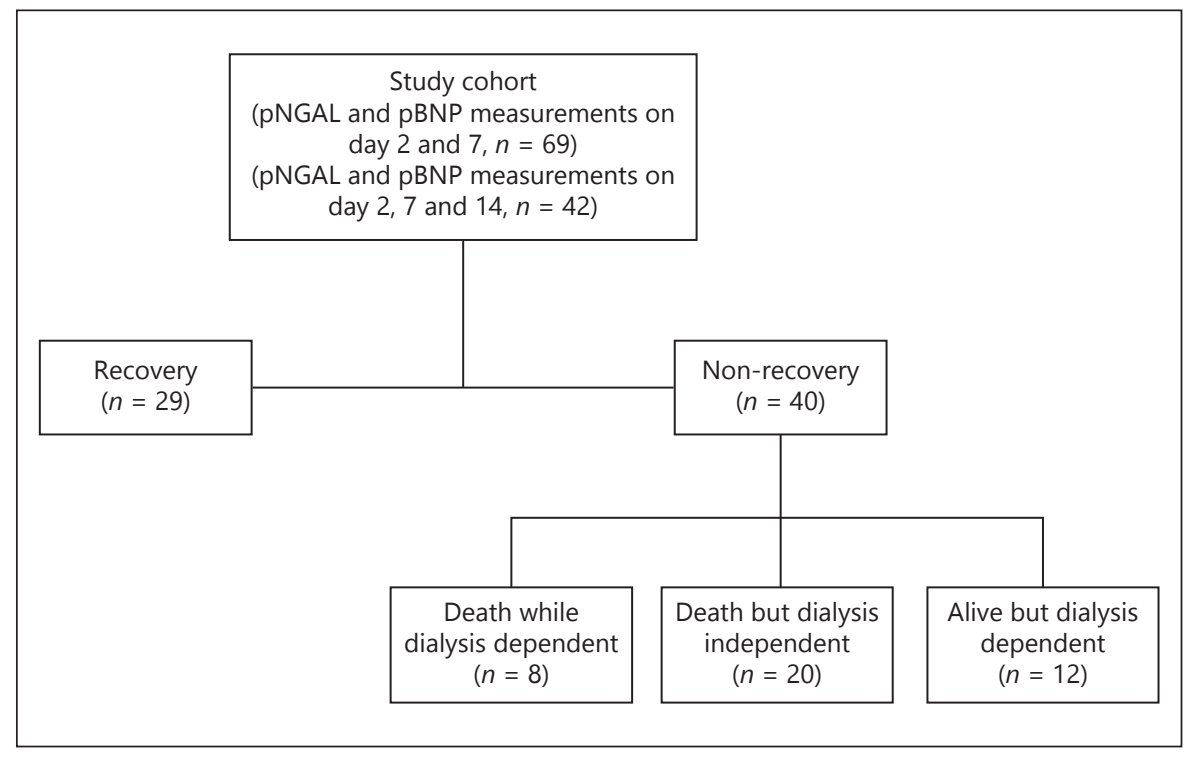

point, while the remaining 40 patients did not recover (12 patients were alive but dialysis dependent, 8 patients died while dialysis dependent, and the remaining 20 subjects were dialysis independent but died; Fig. 1). Baseline clinical and demographic characteristics were shown in Table 1. Patients who recovered from AKI were younger than those who failed to recover (mean age $54.1 \pm 15.5$ vs. $64.6 \pm 14.2, p=0.008$ ). The primary etiology of AKI was ischemia in both groups (overall $84.1 \%$ ), followed by sepsis (59.4\%) and nephrotoxic exposures $(23.2 \%)$. No significant differences between recovery and nonrecovery groups were observed with regard to gender, race, baseline renal function, renal function at the initiation of RRT, causes of AKI, length of ICU and hospital stay, severity of illness scores, and intensity of RRT.

\section{Biomarker Levels and Recovery Status}

No significant differences in pNGAL or pBNP were found at any time point between recovery and nonrecovery patients. No differences in pNGAL were observed in the nonrecovery group between patients who were alive but dialysis dependent, those who died while dialysis dependent and those who died while dialysis independent (online suppl. Fig. 1, 2; for all online suppl. material, see www.karger.com/doi/10.1159/000500423).

\section{Prediction of Renal Recovery by Biomarker \\ Models Alone}

The AUCs for each plasma biomarker for prediction of renal recovery are shown in Table 2. Neither pNGAL nor pBNP alone was good predictors of recovery from $\mathrm{AKI}$ and, interestingly, the best predictive performance was given by pNGAL and pBNP using the largest relative change (AUC 0.59, 95\% CI 0.45-0.74; and 0.59, 95\% CI $0.46-0.73$, respectively). When adding pNGAL to pBNP, the greatest AUC was 0.63 (95\% CI $0.44-0.81$ ) on day 14 and 0.60 (95\% CI 0.47-0.74) for the largest relative change of pNGAL + pBNP. However, we did not find significant differences in predictive accuracy between models with pBNP alone and those including both pBNP and pNGAL. We found similar results in sensitivity analyses, excluding patients who died, but who were dialysis independent or including this group of patients in the recovery group (online suppl. Tables 1,2).

\section{Clinical Risk Prediction Models and Combination of Clinical and Biomarker Models}

We analyzed several clinical risk prediction models previously identified in the Biological Markers of Recovery for the Kidney study and based on age, Charlson comorbidity index and APACHE II [9]. We described the accuracy in predicting renal recovery for each variable individually and then together (Table 3a). The clinical model including only age (model A) had the best AUC in predicting the primary outcome (AUC 0.69, 95\% CI $0.56-$ 0.81) compared to those for Charlson comorbidity index (model B) and APACHE II (model C). Furthermore, the composite clinical model including all variables (model M2) had a similar accuracy (AUC $0.70,95 \%$ CI $0.57-0.84$, $p=0.84)$ compared to model A. Similarly, the model including age and Charlson index (model M1) did not sig- 
Table 1. Baseline clinical characteristics of the study population

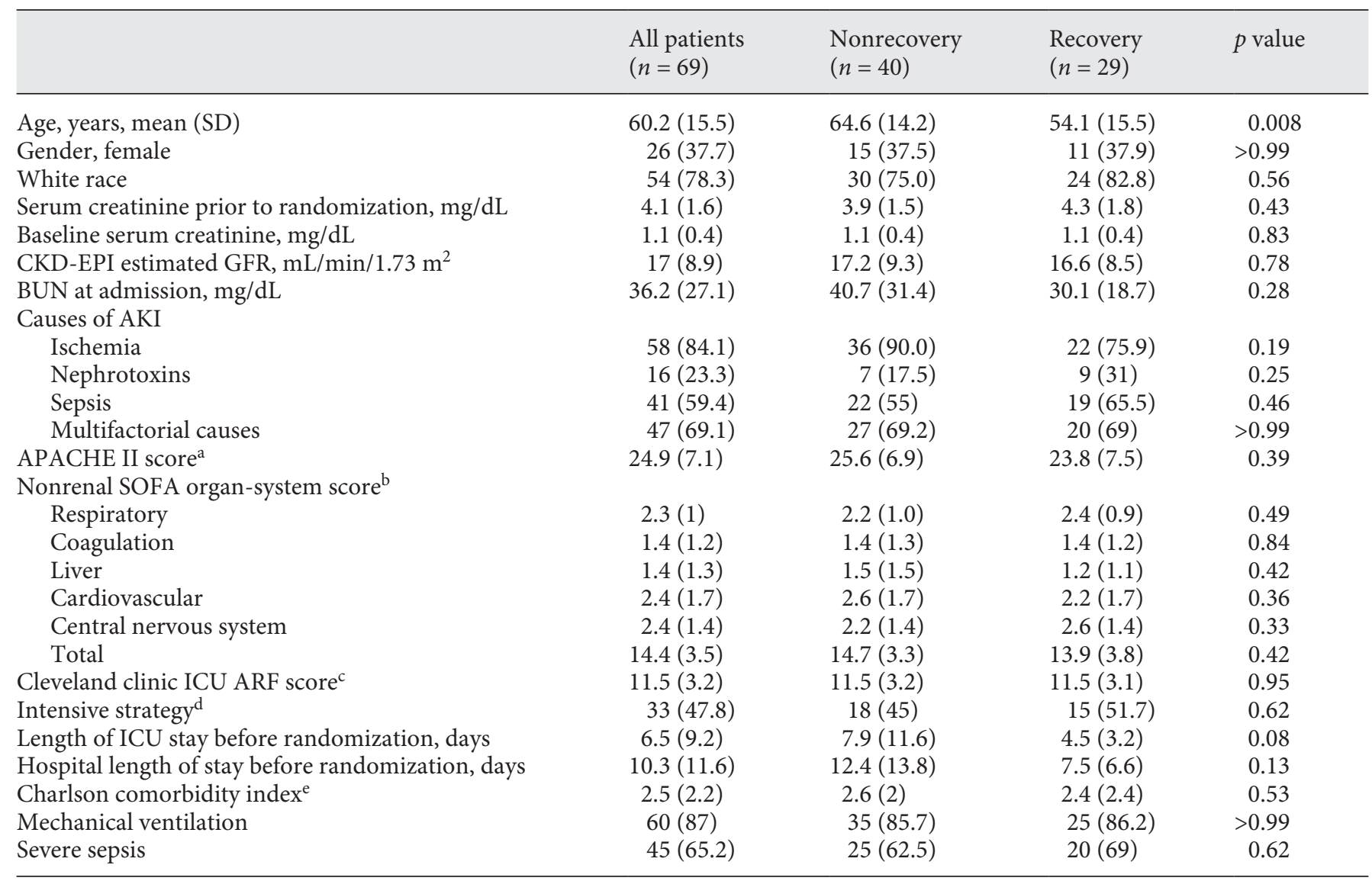

Data presented as mean (SD) or $n(\%)$.

a According to the method of Knaus et al. [13].

${ }^{\mathrm{b}}$ Nonrenal SOFA score was assessed on the first day [14].

${ }^{c}$ According to the method of Thakar et al. [15].

${ }^{\mathrm{d}}$ Intensive strategy: intermittent hemodialysis and sustained low-efficiency dialysis 6 times per week, and continuous venovenous hemodiafiltration at $35 \mathrm{~mL} / \mathrm{kg}$ of body weight per hour [11].

e According to the method of Charlson et al. [16].

AKI, acute kidney injury; BUN, blood urea nitrogen; ICU intensive care unit; APACHE II, Acute Physiology and Chronic Health Evaluation II; SOFA, Sequential Organ Failure Assessment; ARF, acute renal failure.

nificantly differ from the others (AUC 0.70, 95\% CI 0.58$0.83, p=0.61)$.

Next, we analyzed the predictive value of plasma biomarkers when added to the clinical model and compared with the best clinical model (model A). Overall, the combination of the clinical model with biomarkers improved the ability to predict renal recovery, although this difference did not achieve statistical significance. As shown in Table $3 \mathrm{~b}$, we found that on day 2 , the combined model of pNGAL and age had similar accuracy to predict renal recovery (AUC $0.71,95 \%$ CI $0.58-0.83$ ) compared to age alone $($ ROC contrast $p$ value $=0.57$; Fig. $2 a)$.
Similarly, the AUC for the composite model including pNGAL, pBNP, and age on day 14 (AUC 0.75, 95\% CI $0.60-0.91)$ was not significantly higher than that for age alone (ROC contrast $p$ value $=0.62$; Fig. $2 \mathrm{~b}$ ). Moreover, the model combining the largest relative change of pNGAL and $\mathrm{pBNP}$ with age presented a predictive ability (AUC $0.73,95 \%$ CI $0.61-0.85$ ) similar to the clinical model alone ( $\mathrm{ROC}$ contrast $p$ value $=0.35$; Fig. 2c). In sensitivity analysis, when excluding patients who died while dialysis independent from the nonrecovery group, the models including biomarkers had a discrete ability in predicting renal recovery compared 
Table 2. AUC for biomarker models on day 2, 7, 14 and for the largest relative changes for predicting recovery from AKI

\begin{tabular}{|c|c|c|c|c|}
\hline Plasma biomarker & Time point & AUC & $95 \% \mathrm{CI}$ & $p$ value* \\
\hline \multirow[t]{4}{*}{ pNGAL } & Day 2 & 0.54 & $0.41-0.69$ & \\
\hline & Day 7 & 0.50 & $0.36-0.64$ & \\
\hline & Day 14 & 0.55 & $0.37-0.73$ & \\
\hline & Largest relative change $\mathrm{e}^{\#}$ & 0.59 & $0.45-0.74$ & \\
\hline \multirow[t]{4}{*}{$\mathrm{pBNP}$} & Day 2 & 0.50 & $0.36-0.65$ & \\
\hline & Day 7 & 0.58 & $0.45-0.72$ & \\
\hline & Day 14 & 0.59 & $0.41-0.78$ & \\
\hline & Largest relative change $\mathrm{e}^{\#}$ & 0.59 & $0.46-0.73$ & \\
\hline \multirow[t]{3}{*}{ pNGAL and pBNP } & Day 2 & 0.55 & $0.41-0.68$ & 0.89 \\
\hline & Day 14 & 0.63 & $0.44-0.81$ & 0.53 \\
\hline & Largest relative change $\mathrm{e}^{\#}$ & 0.60 & $0.47-0.74$ & 0.86 \\
\hline $\begin{array}{r}\text { \# Largest relative } \\
\text { * Compared witl } \\
\text { Biomarkers analy } \\
\text { AUC, area unde } \\
\text { pBNP, plasma B-typ }\end{array}$ & $\begin{array}{l}\text { in the first } 7 \text { or } 14 \text { days as } \\
\mathrm{L} \text { model on day } 2 \text {, day } 14 \text { a } \\
\text { day } 14 \text { was performed on o } \\
\text { eceiver-operating characte } \\
\text { retic peptide. }\end{array}$ & $\begin{array}{l}\text { o day } 2 . \\
\text { argest re } \\
\text { nts. } \\
\text { pNGA }\end{array}$ & $\begin{array}{l}\text { ange. } \\
\text { a neutrophi }\end{array}$ & -associated lipocalin; \\
\hline
\end{tabular}

Table 3. AUC for clinical risk prediction models alone (a) and for the combination of the best clinical model and biomarker models (b) for each time point for predicting renal recovery

\begin{tabular}{|c|c|c|c|c|c|}
\hline Clinical variables & OR (95\% CI) & Model & AUC & $95 \% \mathrm{CI}$ & $p$ value $^{*}$ \\
\hline Age, years & $0.95(0.92-0.99)$ & A & 0.69 & $0.56-0.81$ & - \\
\hline Charlson comorbidity index & $0.95(0.75-1.2)$ & B & 0.55 & $0.39-0.70$ & - \\
\hline \multirow[t]{3}{*}{ APACHE II } & $0.96(0.90-1.0)$ & $\mathrm{C}$ & 0.56 & $0.42-0.71$ & - \\
\hline & & $M 1=A+C$ & 0.70 & $0.58-0.83$ & 0.61 \\
\hline & & $\mathrm{M} 2+\mathrm{A}+\mathrm{B}+\mathrm{C}$ & 0.70 & $0.57-0.84$ & 0.84 \\
\hline
\end{tabular}

* Compared with model A. II.

AUC, area under the receiver-operating characteristics curve; APACHE II, Acute Physiology and Chronic Health Evaluation

\begin{tabular}{|c|c|c|c|c|}
\hline Time point & Model & AUC & $95 \% \mathrm{CI}$ & $p$ value* \\
\hline \multirow[t]{3}{*}{ Day 2} & Age + pNGAL & 0.71 & $0.58-0.83$ & 0.54 \\
\hline & Age + pBNP & 0.69 & $0.56-0.82$ & 0.86 \\
\hline & Age + pNGAL + pBNP & 0.70 & $0.58-0.83$ & 0.57 \\
\hline \multirow[t]{3}{*}{ Day 14} & Age + pNGAL & 0.74 & $0.58-0.90$ & 0.56 \\
\hline & Age + pBNP & 0.75 & $0.59-0.91$ & 0.74 \\
\hline & Age + pNGAL + pBNP & 0.75 & $0.60-0.91$ & 0.62 \\
\hline \multirow[t]{3}{*}{ Largest relative change ${ }^{\#}$} & Age + pNGAL & 0.70 & $0.57-0.82$ & 0.75 \\
\hline & Age + pBNP & 0.71 & $0.59-0.84$ & 0.48 \\
\hline & Age + pNGAL + pBNP & 0.73 & $0.61-0.85$ & 0.35 \\
\hline
\end{tabular}

\# Largest relative change in the first 14 days as compared to day 2.

* Compared with age alone.

AUC, area under the curve; pNGAL plasma neutrophil gelatinase-associated lipocalin; pBNP, plasma B-type natriuretic peptide. 


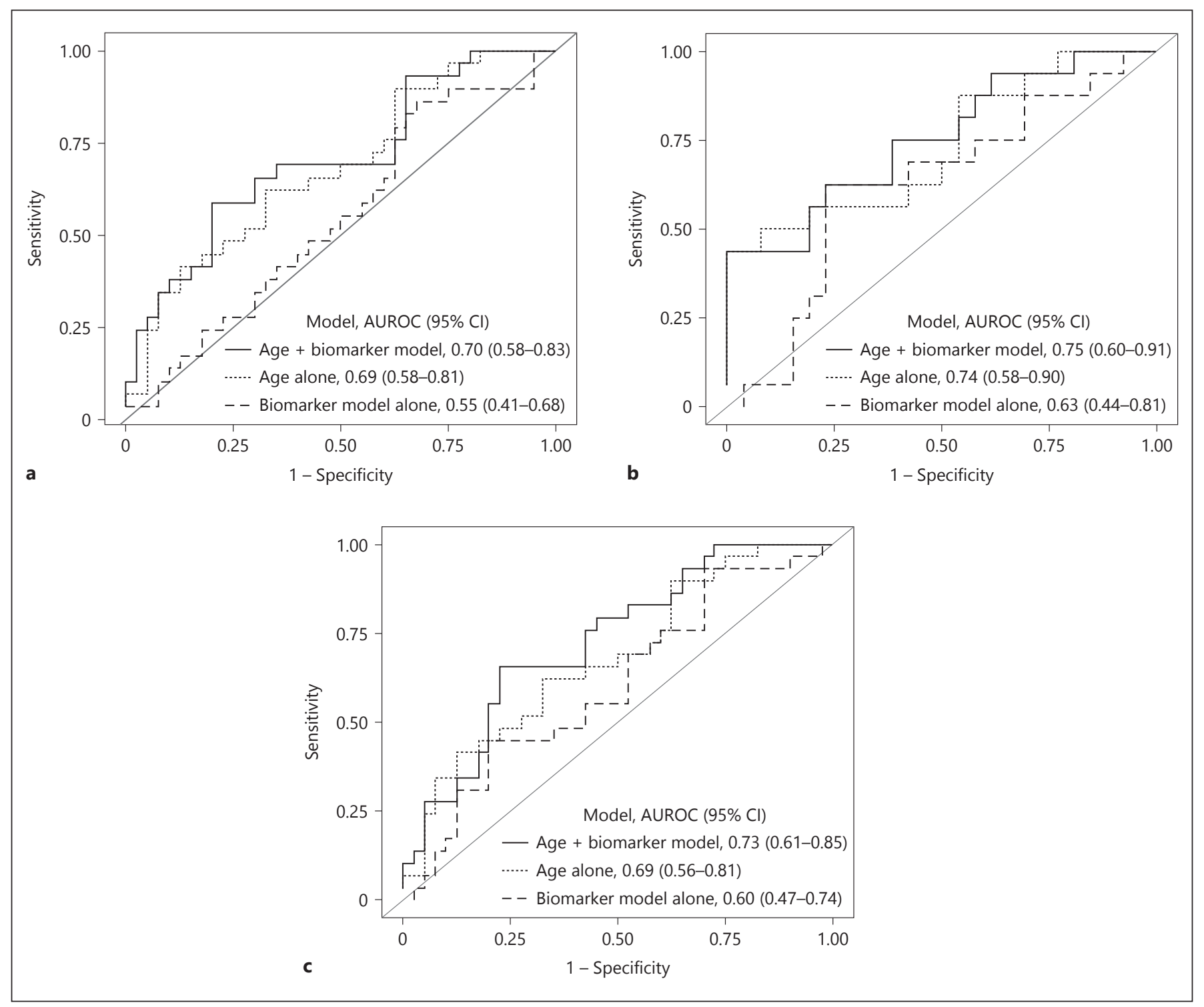

Fig. 2. ROC curves for several prediction models for renal recovery. The data shown represent the comparison between the best biomarker model alone (thick dashed line), the clinical model alone (thin dashed line), and the combined clinical and biomarker model (solid line) on day 2 (a), day 14 (b), and for the largest relative change (c). The clinical prediction model included age alone. The AUC values and 95\% CI are also reported. AUC, area under the ROC curve.

\section{Discussion}

The present study examined one of the most important clinical challenges in critical care medicine, the prediction of recovery after AKI. We found that the integration of plasma biomarkers with clinical parameters can improve the accuracy in identifying subgroups of patients with different likelihood of renal recovery.

Much evidence suggested that patients who fail to recover from AKI have a reduced quality of life and an 
increased risk for chronic disease [3, 17-19]. The multinational Beginning and Ending Supportive Therapy for the kidney study showed that hospital mortality in patients with AKI was very high (60.3\%) and dialysis dependence at hospital discharge was significant as well (13.8\%) [20]. The ATN study described a high percentage of patients who did not recover renal function by day 28 (75.8\% in the intensive RRT group, $72.6 \%$ in the less-intensive RRT group), and only $16 \%$ were discharged to home without dialysis by day 60 [11]. However, our recent data in patients with septic shock showed that those with complete or partial recovery from AKI may have a similar 1-year survival to patients without AKI [21]. These data emphasize the urgent need for new tools to improve recovery and reduce the incidence of negative outcomes in patients with AKI. Clinical parameters (increasing age, comorbidities, severity and duration of $\mathrm{AKI}$, and timing and dose of RRT) have been correlated with renal recovery [2]; however, the ATN study did not describe differences in recovery rate between the intensive and less-intensive RRT strategy groups [11]. In this scenario, a useful approach could be to evaluate certain biomarkers in predicting renal recovery [6]. In this study, we focused on a well-recognized plasma biomarker for renal disease, pNGAL. NGAL has been tested as an early diagnostic tool for AKI [22-25], and it could be an optimal marker of renal recovery. Kusaka et al. [26] evaluated serum NGAL in predicting functional recovery after kidney transplantation and showed that the decrease of NGAL values after transplantation well correlated with the renal recovery and discriminated patients with immediate, slow or delayed graft function. Recently, Liu et al. [27] showed that in 49 adult patients with living donor kidney transplants, serum NGAL on the day of transplantation was an independent predictor of graft function recovery. Previously, we analyzed pNGAL and renal recovery in a post hoc analysis performed as part of the Genetic and Inflammatory Markers of Sepsis study, a multicenter prospective study with patients with community-acquired pneumonia $[5,28]$. In this cohort, pNGAL alone predicted failure to recover with an AUC of 0.74 and a clinical model including age, serum creatinine, pneumonia severity, and nonrenal organ failure score presented a similar AUC (0.78); the AUC did not significantly increase when combining pNGAL and clinical model, but the reclassification of risk of renal recovery significantly improved by $17 \%$ [5].

In this study, we also focused on pBNP to control for cardiovascular effects on recovery. pBNP is a neuropep- tide hormone released from myocytes in response to ventricular stretching; it is a well-known biomarker of cardiac volume and hemodynamics, but it has been recently evaluated as an AKI biomarker, particularly in patients with heart failure [29]. Recently, Howell et al. [30] hypothesized that point-of-care pBNP and pNGAL could be useful for earlier assessment of intravascular volume and renal function in severe burn injury during resuscitation, in order to predict inadequate resuscitation strategies and to define cardiorenal syndrome early. Plasma BNP levels were significantly higher in patients with AKI compared to control patients, as well as in overresuscitated patients $(23.1 \pm 21.9$ vs. $13.9 \pm 13.4 \mathrm{pg} / \mathrm{mL}$, $p<0.001)$ [30].

In sensitivity analyses, when we focused on a specific renal definition for recovery (excluding patients who died while dialysis independent from the nonrecovery group), the accuracy of biomarkers increased and a full model that included biomarkers and age achieved an adequate accuracy in predicting renal recovery at each time point (AUC 0.77 on day 2, 0.76 on day 14, 0.78 using the largest relative change between day 2 and 14). While death and nonrecovery are frequently combined as a composite endpoint (e.g., major adverse kidney events) [31], patients may be interested in understanding the risks of requiring dialysis even if mortality risk is uncertain. There is no agreement about the best definition for renal recovery and the conflicting results about the application of biomarkers among different studies may be related to this issue. Since our analysis focused on a limited cohort, larger studies are needed to confirm the utility of these biomarkers in informing clinical decision-making in critically ill patients [32]. For example, timing cessation of RRT is a very important clinical decision, since unnecessary treatment should be avoided to reduce dialysis complications [7]. In the ATN study, the mean duration of RRT was about $13.4 \pm 9.6$ for the intensive RRT and $12.8 \pm 9.3$ for the less-intensive strategy group [1]. For this analysis, we decided not to focus on RRT duration in predicting renal recovery since patients who do not recover obviously undergo a longer RRT duration. Furthermore, since failure to recover is associated with worse outcomes (chronic kidney disease), these patients can be targeted for closer follow-up and for specific interventions (placement of permanent vascular access and kidney transplantation for those who develop end-stage renal disease).

Our study has some important limitations. First, sample size was limited by the availability of biomarker measurements, so we did not explore comparison of biomarker levels between complete and partial recovery. Second, 
this is an ancillary analysis from the ATN study and the data are relatively old (between 2003 and 2007), although samples have been maintained at $-80^{\circ}$. Also, we did not have long-term follow-up data. Third, we only measured 2 biomarkers. Other serum and urinary biomarkers, including cell-cycle arrest biomarkers $[33,34]$, might also be useful for predicting AKI and renal recovery. Moreover, we did not analyze the potential differences in NGAL removal between different dialysis techniques. Finally, the ATN study did not collect data on fluid balance and this could limit the interpretation of $\mathrm{pBNP}$ data since prior literature has described its relevance in the setting of fluid overload.

\section{Conclusions}

In conclusion, our results demonstrate that models including plasma NGAL and BNP and clinical information can provide reasonably robust prediction of patients more or less likely to recover after AKI requiring RRT, especially when focusing on a specific renal definition for recovery. Additional studies will be required to determine whether these results can be incorporated into clinical protocols to improve patient care.

\section{Trial Registration}

The present study was a prospective cohort study conducted as an ancillary analysis to the ATN study. The Institutional Review Boards at all participating sites approved the parent study, and written informed consent was obtained from all participants or their proxies. NCT number: NCT00076219, first posted January 19, 2004 (retrospectively registered).

\section{Acknowledgements}

Not applicable.

\section{Statement of Ethics}

The present study was a prospective cohort study conducted as an ancillary analysis to the ATN study. The Institutional Review Boards at all participating sites approved the parent study, and written informed consent was obtained from all participants or their proxies.

\section{Disclosure Statement}

J.A.K. and R.M. report grant support from Bioporto. M.F. reports consulting fees from Bioporto.

\section{Funding Sources}

This work was funded by institutional grants. The content is solely the responsibility of the authors. BioMaRK was supported by a grant (R01DK070910) from the National Institute of Diabetes, and Digestive, and Kidney Diseases (NIDDK). The content of this paper is solely the responsibility of the authors and does not necessarily represent the official views of NIDDK or NIH. The VA/NIH ATN study was supported by the Cooperative Studies Program of the Department of Veterans Affairs Office of Research and Development (CSP \# 530$)$ and by NIDDK by interagency agreement Y1DK-3508.

\section{Author Contributions}

M.F., F.A.T., and J.A.K. developed the research idea and study design for the present analysis and wrote the paper. R.M. provided critical review and revised the paper.

\section{References}

1 Koraishy FM, Coca SG. Can we predict recovery from severe acute kidney injury with biomarkers? Semin Dial. 2014 May-Jun;27(3): 236-9.

2 Godin M, Macedo E, Mehta RL. Clinical determinants of renal recovery. Nephron Clin Pract. 2014;127(1-4):25-9.

3 Goldstein SL, Chawla L, Ronco C, Kellum JA. Renal recovery. Crit Care. 2014 Jan;18(1):301.

4 Kellum JA. How can we define recovery after acute kidney injury? Considerations from epidemiology and clinical trial design. Nephron Clin Pract. 2014;127(1-4):81-8.

5 Srisawat N, Murugan R, Lee M, Kong L, Carter M, Angus DC, et al.; Genetic and Inflammatory Markers of Sepsis (GenIMS) Study
Investigators. Plasma neutrophil gelatinaseassociated lipocalin predicts recovery from acute kidney injury following community-acquired pneumonia. Kidney Int. 2011 Sep; 80(5):545-52.

6 Srisawat N, Murugan R, Kellum JA. Repair or progression after AKI: a role for biomarkers? Nephron Clin Pract. 2014;127(1-4):185-9.

7 Endre ZH. Recovery from acute kidney injury: the role of biomarkers. Nephron Clin Pract. 2014;127(1-4):101-5.

8 Coca SG, Yalavarthy R, Concato J, Parikh CR. Biomarkers for the diagnosis and risk stratification of acute kidney injury: a systematic review. Kidney Int. 2008 May;73(9): 1008-16.
9 Srisawat N, Wen X, Lee M, Kong L, Elder M, Carter M, et al. Urinary biomarkers and renal recovery in critically ill patients with renal support. Clin J Am Soc Nephrol. 2011 Aug; 6(8):1815-23.

10 Chawla LS, Amdur RL, Shaw AD, Faselis C, Palant CE, Kimmel PL. Association between $\mathrm{AKI}$ and long-term renal and cardiovascular outcomes in United States veterans. Clin J Am Soc Nephrol. 2014 Mar;9(3):448-56.

11 Palevsky PM, Zhang JH, O'Connor TZ, Chertow GM, Crowley ST, Choudhury D, et al.; VA/NIH Acute Renal Failure Trial Network. Intensity of renal support in critically ill patients with acute kidney injury. $\mathrm{N}$ Engl J Med. 2008 Jul;359(1):7-20. 
12 Singer M, Deutschman CS, Seymour CW, Shankar-Hari M, Annane D, Bauer M, et al. The Third International Consensus Definitions for Sepsis and Septic Shock (Sepsis-3). JAMA. 2016 Feb;315(8):801-10.

13 Knaus WA, Wagner DP, Draper EA, Zimmerman JE, Bergner M, Bastos PG, et al. The APACHE III prognostic system. Risk prediction of hospital mortality for critically ill hospitalized adults. Chest. 1991 Dec;100(6): 1619-36.

14 Vincent JL, Moreno R, Takala J, Willatts S, De Mendonça A, Bruining $\mathrm{H}$, et al. The SOFA (Sepsis-related Organ Failure Assessment) score to describe organ dysfunction/failure. On behalf of the Working Group on SepsisRelated Problems of the European Society of Intensive Care Medicine. Intensive Care Med. 1996 Jul;22(7):707-10.

15 Thakar CV, Arrigain S, Worley S, Yared JP, Paganini EP. A clinical score to predict acute renal failure after cardiac surgery. J Am Soc Nephrol. 2005 Jan;16(1):162-8.

16 Charlson ME, Pompei P, Ales KL, MacKenzie CR. A new method of classifying prognostic comorbidity in longitudinal studies: development and validation. J Chronic Dis. 1987; 40(5):373-83

17 Doyle JF, Forni LG. Long-Term Follow-up of Acute Kidney Injury. Crit Care Clin. 2015 Oct;31(4):763-72.

18 Kellum JA. Diagnostic Criteria for Acute Kidney Injury: present and Future. Crit Care Clin. 2015 Oct;31(4):621-32.

19 Kidney Disease: Improving Global Outcomes (KDIGO) Acute Kidney Injury Work Group. KDIGO Clinical Practice Guideline for Acute Kidney Injury. Kidney Inter. 2012;2(2 Suppl): $1-138$.

20 Uchino S, Kellum JA, Bellomo R, Doig GS, Morimatsu $\mathrm{H}$, Morgera S, et al.; Beginning and Ending Supportive Therapy for the Kidney (BEST Kidney) Investigators. Acute renal failure in critically ill patients: a multina- tional, multicenter study. JAMA. 2005 Aug; 294(7):813-8.

21 Kellum JA, Chawla LS, Keener C, Singbartl K, Palevsky PM, Pike FL, et al.; ProCESS and ProGReSS-AKI Investigators. The Effects of Alternative Resuscitation Strategies on Acute Kidney Injury in Patients with Septic Shock. Am J Respir Crit Care Med. 2016 Feb;193(3): 281-7.

22 Ling W, Zhaohui N, Ben H, Leyi G, Jianping L, Huili D, et al. Urinary IL-18 and NGAL as early predictive biomarkers in contrast-induced nephropathy after coronary angiography. Nephron Clin Pract. 2008;108(3):c17681.

23 Lipcsey M, Hayward P, Haase M, HaaseFielitz A, Eastwood G, Peck L, et al. Neutrophil gelatinase-associated lipocalin after off pump versus on pump coronary artery surgery. Biomarkers. 2014 Feb;19(1):22-8.

24 Mishra J, Dent C, Tarabishi R, Mitsnefes MM, Ma Q, Kelly C, et al. Neutrophil gelatinaseassociated lipocalin (NGAL) as a biomarker for acute renal injury after cardiac surgery. Lancet. 2005 Apr;365(9466):1231-8.

25 Huang CY, Shih CC, Chung K, Kao KC, Wu HP. Predictive value of plasma neutrophil gelatinase-associated lipocalin for acute renal failure in patients with severe sepsis. J Chin Med Assoc. 2016 Aug;79(8):428-34.

26 Kusaka M, Iwamatsu F, Kuroyanagi Y, Nakaya $M$, Ichino $M$, Marubashi S, et al. Serum neutrophil gelatinase associated lipocalin during the early postoperative period predicts the recovery of graft function after kidney transplantation from donors after cardiac death. J Urol. 2012 Jun; 187(6): 2261-7.

27 Liu Y, Li HX, Ying ZW, Guo JJ, Cao CY, Jia W, et al. Serum Neutrophil Gelatinase-Associated Lipocalin and Cystatin C for Assessing Recovery of Graft Function in Patients Undergoing Living-Donor Kidney Transplantation. Clin Lab. 2016;62(1-2):155-63.
28 Kellum JA, Kong L, Fink MP, Weissfeld LA, Yealy DM, Pinsky MR, et al.; GenIMS Investigators. Understanding the inflammatory cytokine response in pneumonia and sepsis: results of the Genetic and Inflammatory Markers of Sepsis (GenIMS) Study. Arch Intern Med. 2007 Aug;167(15):1655-63.

29 Palazzuoli A, Ruocco G, Pellegrini M, De Gori C, Del Castillo G, Franci B, et al. Comparison of Neutrophil Gelatinase-Associated Lipocalin Versus B-Type Natriuretic Peptide and Cystatin C to Predict Early Acute Kidney Injury and Outcome in Patients With Acute Heart Failure. Am J Cardiol. 2015 Jul;116(1): 104-11.

30 Howell E, Sen S, Palmieri T, Godwin Z, Bockhold J, Greenhalgh D, et al. Point-of-care Btype natriuretic peptide and neutrophil gelatinase-associated lipocalin measurements for acute resuscitation: a pilot study. J Burn Care Res. 2015 Mar-Apr;36(2):e26-33.

31 Palevsky PM, Molitoris BA, Okusa MD, Levin A, Waikar SS, Wald R, et al. Design of clinical trials in acute kidney injury: report from an NIDDK workshop on trial methodology. Clin J Am Soc Nephrol. 2012 May;7(5):844-50.

32 Chawla LS, Bellomo R, Bihorac A, Goldstein SL, Siew ED, Bagshaw SM, et al.; Acute Disease Quality Initiative Workgroup 16. Acute kidney disease and renal recovery: consensus report of the Acute Disease Quality Initiative (ADQI) 16 Workgroup. Nat Rev Nephrol. 2017 Apr;13(4):241-57.

33 Bihorac A, Chawla LS, Shaw AD, Al-Khafaji A, Davison DL, Demuth GE, et al. Validation of cell-cycle arrest biomarkers for acute kidney injury using clinical adjudication. Am J Respir Crit Care Med. 2014 Apr;189(8):9329.

34 Kashani K, Al-Khafaji A, Ardiles T, Artigas A, Bagshaw SM, Bell M, et al. Discovery and validation of cell cycle arrest biomarkers in human acute kidney injury. Crit Care. 2013 Feb; 17(1):R25. 